\title{
Solar abundance of manganese: a case for near Chandrasekhar-mass Type la supernova progenitors
}

\author{
Ivo R. Seitenzahl ${ }^{1,2}$, Gabriele Cescutti ${ }^{3}$, Friedrich K. Röpke ${ }^{1}$, Ashley J. Ruiter ${ }^{2}$, and Rüdiger Pakmor ${ }^{4}$ \\ ${ }^{1}$ Institut für Theoretische Physik und Astrophysik, Universität Würzburg, Campus Hubland Nord, Emil-Fischer-Str. 31, \\ 97074 Würzburg, Germany \\ e-mail: iseitenzahl@astro.uni-wuerzburg.de \\ 2 Max-Planck-Institut für Astrophysik, Karl-Schwarzschild-Str. 1, 85741 Garching, Germany \\ ${ }^{3}$ Leibniz-Institut für Astrophysik Potsdam (AIP), An der Sternwarte 16, 14482 Potsdam, Germany \\ ${ }^{4}$ Heidelberger Institut für Theoretische Studien, Schloss-Wolfsbrunnenweg 35, 69118 Heidelberg, Germany
}

Received 3 September 2013 / Accepted 1 October 2013

\section{ABSTRACT}

\begin{abstract}
Context. Manganese is predominantly synthesised in Type Ia supernova (SN Ia) explosions. Owing to the entropy dependence of the Mn yield in explosive thermonuclear burning, SNe Ia involving near Chandrasekhar-mass $\left(\mathrm{M}_{\mathrm{Ch}}\right)$ white dwarfs (WDs) are predicted to produce Mn-to-Fe ratios that significantly exceed those of SN Ia explosions involving sub-Chandrasekhar mass primary WDs. Of all current supernova explosion models, only SN Ia models involving near- $\mathrm{M}_{\mathrm{Ch}}$ WDs produce $[\mathrm{Mn} / \mathrm{Fe}] \gtrsim 0.0$.

Aims. Using the specific yields for competing SN Ia scenarios, we aim to constrain the relative fractions of exploding near-M $\mathrm{M}_{\mathrm{Ch}}$ to sub-M $\mathrm{M}_{\mathrm{Ch}}$ primary WDs in the Galaxy.

Methods. We extract the Mn yields from three-dimensional thermonuclear supernova simulations that refer to different initial setups and progenitor channels. We then compute the chemical evolution of $\mathrm{Mn}$ in the solar neighborhood, assuming SNe Ia are made up of different relative fractions of the considered explosion models.

Results. We find that due to the entropy dependence of freeze-out yields from nuclear statistical equilibrium, $[\mathrm{Mn} / \mathrm{Fe}] \mathrm{depends}$ strongly on the mass of the exploding WD, with near- $\mathrm{M}_{\mathrm{Ch}}$ WDs producing substantially higher $[\mathrm{Mn} / \mathrm{Fe}]$ than sub-M $\mathrm{M}_{\mathrm{Ch}} \mathrm{WDs}_{\mathrm{S}}$ Of all nucleosynthetic sources potentially influencing the chemical evolution of $\mathrm{Mn}$, only explosion models involving the thermonuclear incineration of near- $\mathrm{M}_{\mathrm{Ch}}$ WDs predict solar or super-solar $[\mathrm{Mn} / \mathrm{Fe}]$. Consequently, we find in our chemical evolution calculations that the observed $[\mathrm{Mn} / \mathrm{Fe}]$ in the solar neighborhood at $[\mathrm{Fe} / \mathrm{H}] \gtrsim 0.0$ cannot be reproduced without near- $\mathrm{M}_{\mathrm{Ch}} \mathrm{SN}$ Ia primaries. Assuming that $50 \%$ of all SNe Ia stem from explosive thermonuclear burning in near-M $\mathrm{M}_{\mathrm{Ch}}$ WDs results in a good match to data.
\end{abstract}

Key words. supernovae: general - nuclear reactions, nucleosynthesis, abundances - Galaxy: abundances - Galaxy: evolution

\section{Introduction}

There is general consensus that thermonuclear explosions of carbon-oxygen WDs are the underlying physical process leading to Type Ia supernova (SN Ia) explosions (for a recent review on SNe Ia see, for instance, Hillebrandt et al. 2013). In spite of this general agreement on the basic underlying physical picture, neither the exact explosion mechanism(s) nor the formation channel(s) of binary stellar evolution leading up to the explosion have reached a consensus model.

Loosely speaking, two main evolutionary scenarios have emerged. In the single-degenerate scenario (SDS) first described by Whelan \& Iben (1973), a WD accretes mass from a stellar companion until it explodes following the onset of a carbon fusion runaway as it approaches the Chandrasekhar-mass $\left(\mathrm{M}_{\mathrm{Ch}}\right)$ limit. Recent multi-dimensional simulations of explosions of near- $\mathrm{M}_{\mathrm{Ch}}$ WDs include pure deflagration (e.g. Röpke et al. 2007; Jordan et al. 2012b; Ma et al. 2013; Fink et al. 2013), deflagration-to-detonation transition (e.g. Gamezo et al. 2005; Röpke \& Niemeyer 2007; Bravo \& García-Senz 2008; Kasen et al. 2009; Seitenzahl et al. 2011, 2013), pulsational reverse detonation (e.g. Bravo \& García-Senz 2009), and variants of gravitational confined detonation models (e.g. Plewa 2007; Meakin et al. 2009; Jordan et al. 2012a). In the doubledegenerate scenario (DDS) first proposed by Iben \& Tutukov (1984) and Webbink (1984), the progenitor system is a binary system of two WDs. For sufficiently close binaries, the emission of gravitational waves will lead to orbital decay, potentially resulting in a thermonuclear explosion triggered by the merger of the two WDs. Proposed explosion mechanisms in the DDS can be divided into two categories, depending on the existence of an accretion torus.

(1) Although it is generally believed that accretion from the thick disc around the primary (e.g. Tutukov \& Yungelson 1979; Mochkovitch \& Livio 1990) leads to its collapse to a neutron star (e.g. Nomoto \& Kondo 1991; Dessart et al. 2006; Yoon et al. 2007) following its transformation to an O-Ne-Mg core (Saio \& Nomoto 1985; Timmes 1994; Saio \& Nomoto 1998), Piersanti et al. (2003a,b) and Saio \& Nomoto (2004) argue that for rapidly rotating primaries, central carbon ignition may be possible. The latter case would result in a near- $\mathrm{M}_{\mathrm{Ch}} \mathrm{SN}$ Ia event, with the same potential explosion mechanisms listed above.

(2) Recent multi-dimensional hydrodynamical simulations have shown that an accretion disc need not form, and the resulting violent merger of the two WDs may lead to a detonation in the primary (Pakmor et al. 2010, 2011, 2012; Dan et al. 2011; Raskin et al. 2012). In this violent merger model, the explosion is essentially driven by a pure detonation of a nearly hydrostatic sub-M $\mathrm{M}_{\mathrm{Ch}}$ WD.

From the point of view of explosion modelling, the important question is whether the primary WD is near- $\mathrm{M}_{\mathrm{Ch}}$ (resulting from 
the SDS or mergers with accretion from a torus) or significantly sub- $\mathrm{M}_{\mathrm{Ch}}$ (from violent mergers or double detonations in Heaccreting systems, e.g. Woosley \& Weaver 1994). Mazzali et al. (2007) argue for the former case, while Stritzinger et al. (2006) support the latter. We show that the two possibilities lead to significant differences in the $\mathrm{Mn}$-to-Fe production ratio, and we argue that a significant fraction of Galactic SNe Ia must arise from explosions of near- $\mathrm{M}_{\mathrm{Ch}}$ WDs. We continue by analyzing the impact of the difference in Mn on chemical evolution models and comparing the results to observational data on $\mathrm{Mn}$ abundances in the Sun and in Galactic stars.

\section{Nucleosynthesis of $\mathrm{Mn}$ in $\mathrm{SN}$ la}

A key focus of this work is on the production of manganese in explosive nucleosynthesis. Mn (atomic number 25) has only one stable isotope, ${ }^{55} \mathrm{Mn}$. Most of the ${ }^{55} \mathrm{Mn}$ produced in thermonuclear explosive burning is synthesised as ${ }^{55} \mathrm{Co}$ (e.g. Truran et al. 1967), which then decays via ${ }^{55} \mathrm{Fe}$ to the stable ${ }^{55} \mathrm{Mn}$. The two main nucleosynthetic processes synthesising ${ }^{55} \mathrm{Co}$, hence $\mathrm{Mn}$, are "normal" freeze-out from nuclear statistical equilibrium (NSE) and incomplete Si-burning. For freeze-out from NSE to be "normal" as opposed to "alpha-rich", the mass fraction of ${ }^{4} \mathrm{He}$ has to remain rather low during the freeze-out phase ( $\lesssim 1$ per cent according to Woosley et al. 1973). For explosive nuclear burning this is the case at relatively high density $\left(\rho \gtrsim 2 \times 10^{8} \mathrm{~g} \mathrm{~cm}^{-3}\right.$, see Thielemann et al. 1986; Bravo \& Martínez-Pinedo 2012), which implies relatively low entropy. At lower density, the ${ }^{55}$ Co present in NSE is readily destroyed during the alpha-rich freeze-out via ${ }^{55} \mathrm{Co}(p, \gamma){ }^{56} \mathrm{Ni}$ (see Jordan et al. 2003), resulting in a much lower final $[\mathrm{Mn} / \mathrm{Fe}]$. We note that a recent study has shown that the ${ }^{55} \mathrm{Co}$ to ${ }^{56} \mathrm{Ni}$ production ratio is rather insensitive to nuclear reaction rate uncertainties (Parikh et al. 2013).

To put this critical density into context, note that the mass of a cold WD $\left(Y_{\mathrm{e}}=0.5\right)$ in hydrostatic equilibrium with central density $\rho_{\mathrm{c}}=2 \times 10^{8} \mathrm{~g} \mathrm{~cm}^{-3}$ is $M=1.22 M_{\odot}$. Only explosions of near- $\mathrm{M}_{\mathrm{Ch}}$ WDs involve densities high enough to result in "normal" freeze-out from NSE. Violent mergers (Pakmor et al. 2012), as well as sub- $\mathrm{M}_{\mathrm{Ch}}$ double detonations (e.g. Fink et al. 2010; Kromer et al. 2010) of typical SN Ia brighness have primary core masses below $1.2 M_{\odot}$ (Sim et al. 2010; Ruiter et al. 2011). We therefore have a robust, physical reason for the large difference in $[\mathrm{Mn} / \mathrm{Fe}]$. Delayed-detonation models, which undergo significant thermonuclear explosive burning at densities above $\rho \gtrsim 2 \times 10^{8} \mathrm{~g} \mathrm{~cm}^{-3}$ will have an enhanced production of Mn from the contribution of "normal" freeze-out from NSE, which is not the case for violent merger or doubledetonation models. This division between "normal" and "alpharich" freeze-out is also the reason for the predicted differences of the late-time bolometric light curves (Seitenzahl et al. 2009; Röpke et al. 2012).

We note that for very neutron-rich environments, ${ }^{55} \mathrm{Mn}$ could also be directly synthesised. Therefore, it is natural to ask the question of whether gravitational settling of ${ }^{22} \mathrm{Ne}$ in sub- $\mathrm{M}_{\mathrm{Ch}}$ WDs can significantly affect our main point that $[\mathrm{Mn} / \mathrm{Fe}]$ for $\mathrm{SNe}$ Ia resulting from these objects is significantly sub-solar. In contrast to canonical ignition in near- $\mathrm{M}_{\mathrm{Ch}}$ WDs, convective burning is not expeced to precede the explosion here. The potential effects of concentrating neutron-rich material near the WD's core are therefore possible in principle. For gravitational settling to play a role, i) the sub- $\mathrm{M}_{\mathrm{Ch}} \mathrm{WD}$ has to remain liquid; and ii) sufficient time must pass to allow for appreciable ${ }^{22} \mathrm{Ne}$ to fall from low- to high-density regions where iron-group nucleosynthesis occurs. That the sub- $\mathrm{M}_{\mathrm{Ch}}$ primary WD in a DDS system remains liquid for the ${ }^{22} \mathrm{Ne}$ to settle is already unlikely, since for cooling and non-accreting WDs the ${ }^{22} \mathrm{Ne}$ settling time scale $\left(t_{\mathrm{s}}\right)$ is longer than the crystallisation time scale in the core (Bildsten $\&$ Hall 2001). Even if the WD were to remain liquid, the relevant time scales are too long to significantly affect our conclusions. For example, for a hot $\left(T=10^{8} \mathrm{~K}\right) 1.2 M_{\odot} \mathrm{WD}, t_{\mathrm{s}} \approx 5 \mathrm{Gyr}$, and for a cold $\left(T=10^{6} \mathrm{~K}\right) 1.2 M_{\odot} \mathrm{WD}, t_{\mathrm{s}} \approx 23 \mathrm{Gyr}$ (Bravo et al. 1992). Furthermore, the settling time scale $t_{\mathrm{s}}$ is increasing strongly with decreasing WD mass (e.g. Bildsten \& Hall 2001). Consequently, less massive WDs around 1.0 $M_{\odot}$ would show even less of an effect. Since most SNe Ia have much shorter delay times (e.g. Maoz \& Mannucci 2012), we expect that gravitational settling of ${ }^{22} \mathrm{Ne}$ will not change our conclusions.

\section{Galactic chemical evolution of Mn}

Observational data show that halo stars have an average abundance ratio for $[\mathrm{Mn} / \mathrm{Fe}] \sim-0.5$ (see Sobeck et al. 2006), providing a strong indication that SNe II produce a sub-solar ratio of $\mathrm{Mn}$ to Fe. Theoretical nucleosynthesis calculations of massive stars agree with these observational findings; most of the models (e.g. Woosley \& Weaver 1995; Limongi \& Chieffi 2003; Nomoto et al. 2006) predict $[\mathrm{Mn} / \mathrm{Fe}]$ yields that are typically three times lower than the one observed in the Sun. The solar value for the mass ratio of Fe to $\mathrm{Mn}$ can be computed from the photospheric abundances (Grevesse et al. 2010) by assuming the same mean atomic weights observed on Earth. Assuming uncorrelated errors, we obtain $\mathrm{Fe} / \mathrm{Mn}=119 \pm 15$ for the elemental mass ratio.

$\mathrm{SNe}$ Ia enrich the interstellar medium with a time delay compared to the first core-collapse $\mathrm{SNe}$, which means that they did not significantly affect the chemical evolution in the solar vicinity until $[\mathrm{Fe} / \mathrm{H}] \sim-1.0$ (see e.g. Matteucci \& Greggio 1986). Indeed, from around this metallicity, $[\mathrm{Mn} / \mathrm{Fe}]$ derived from observed stellar abundances displays a strong increase (e.g. Gratton \& Sneden 1988, 1991). Although Feltzing et al. (2007) invoke strongly metallicity-dependent SNe II Mn yields, the rise in $[\mathrm{Mn} / \mathrm{Fe}]$ for $[\mathrm{Fe} / \mathrm{H}] \gtrsim-1.0$ to the value observed in the Sun is typically attributed to the nucleosynthesis contribution of SNe Ia (e.g. Gratton 1989; Timmes et al. 1995; François et al. 2004; Cescutti et al. 2008; Kobayashi et al. 2006; Kobayashi \& Nomoto 2009; Kobayashi et al. 2011).

We perform chemical evolution calculations (see Sect. 4) that only differ in the yields assumed for SNe Ia (see Sect. 3.1). Our model for the solar vicinity, which is essentially the same as adopted in Cescutti et al. (2008), is based on the model introduced by Chiappini et al. (1997) (called "two infall model"). For all cases, we use the same delay time distribution (DTD; Greggio \& Renzini 1983), although we are aware that this is a simplistic approach. Assuming a different DTD for, say, the merger scenario from analytical formalisms (e.g. as in Greggio 2005) or binary evolution calculations (Ruiter et al. 2009) could modify the trend obtained by our chemical evolution model. Examples of the sensitivity on the DTD can be found in Matteucci et al. (2009) for the case of $[\mathrm{O} / \mathrm{Fe}]$ and in Kobayashi \& Nomoto (2009). However, assuming yields for SNe Ia lower than solar will always result in a $\mathrm{Mn}$ to $\mathrm{Fe}$ ratio below the solar value, independent of the assumed DTD. For the contribution of massive star explosions we assume the metallicity-dependent yields calculated by Woosley \& Weaver (1995). We note that these yields do not substantially differ from the yields calculated by other groups (see e.g. Limongi \& Chieffi 2003; Nomoto et al. 2006; Kobayashi et al. 2011). We did not include the contribution of low- and intermediate-mass stars here (e.g. Pignatari et al. 2013), 
Table 1. $[\mathrm{Mn} / \mathrm{Fe}]$ yields for selected thermonuclear (Ia), core collapse (II), and hypernova (HN) models of solar-metallicity progenitors.

\begin{tabular}{|c|c|c|c|c|}
\hline Model name & SN type & Masses & {$[\mathrm{Mn} / \mathrm{Fe}]$} & Ref \\
\hline N100 & Ia & near- $\mathrm{M}_{\mathrm{Ch}}$ & 0.33 & (1) \\
\hline N5def & Ia & near- $M_{C h}$ & 0.36 & (2) \\
\hline N150def & Ia & near- $\mathrm{M}_{\mathrm{Ch}}$ & 0.42 & (2) \\
\hline W7 & Ia & near- $\mathrm{M}_{\mathrm{Ch}}$ & 0.15 & (3) \\
\hline W7 & Ia & near- $M_{C h}$ & 0.02 & (4) \\
\hline $1.1 \_0.9$ & Ia & sub-M $\mathrm{M}_{\mathrm{Ch}}$ & $-0.15^{a}$ & (5) \\
\hline $1.0 \overline{6} M_{\odot}$ & Ia & sub-M $\mathrm{M}_{\mathrm{Ch}}$ & $-0.13^{a}$ & (6) \\
\hline $\mathrm{WW} 95 \mathrm{~B}^{b}$ & II & $11<M / M_{\odot}<40$ & $-0.15^{c}$ & (7) \\
\hline $\mathrm{LC} 03 \mathrm{D}^{d}$ & II & $13<M / M_{\odot}<35$ & $-0.27^{c}$ & (8) \\
\hline N06 & $\mathrm{II}+\mathrm{HN}$ & $13<M / M_{\odot}<40$ & $-0.31^{c}$ & (9) \\
\hline
\end{tabular}

Notes. Only models of near- $\mathrm{M}_{\mathrm{Ch}} \mathrm{SNe}$ Ia predict $[\mathrm{Mn} / \mathrm{Fe}] \geq 0.0 .{ }^{(a)}$ The given reference is for the explosion model; the respective $[\mathrm{Mn} / \mathrm{Fe}]$ yields are published here for the first time, assuming that the main sequence progenitor had a solar metallicity (Asplund et al. 2009) and primary $\mathrm{C}, \mathrm{N}, \mathrm{O}$ was converted to ${ }^{22} \mathrm{Ne}$ during core He-burning. ${ }^{(b)}$ We use model B for $M \geq 30 M_{\odot} \cdot{ }^{(c)}$ Weighted with a Salpeter IMF. ${ }^{(d)}$ We use model sequence $\mathrm{D}$ throughout.

References. (1) Seitenzahl et al. (2013); (2) Fink et al. (2013); (3) Iwamoto et al. (1999); (4) Maeda et al. (2010); (5) Pakmor et al. (2012); (6) Ruiter et al. (2013); (7) Woosley \& Weaver (1995); (8) Limongi \& Chieffi (2003); (9) Nomoto et al. (2006).

since they do not produce or destroy enough Mn or Fe to significantly affect our results.

\subsection{SN la yield data}

We use different yields for near- $\mathrm{M}_{\mathrm{Ch}}$ and sub- $\mathrm{M}_{\mathrm{Ch}}$ explosion models. As our main representative for near- $\mathrm{M}_{\mathrm{Ch}}$ primaries (often likened to the SDS), we use the N100 model of a delayed detonation from Seitenzahl et al. (2013). For sub-M $\mathrm{M}_{\mathrm{Ch}}$ primaries, we use the violent merger model of two WDs with 1.1 and $0.9 M_{\odot}$ published in Pakmor et al. (2012), which can also be thought of as a representative of the DDS. We have chosen these two models since they produce rather typical ${ }^{56} \mathrm{Ni}$ masses of $\sim 0.6 M_{\odot}$ and have already been compared in their optical (Röpke et al. 2012) and gamma-ray (Summa et al. 2013) emission. Due to a significant difference in central density, the production of $\mathrm{Mn}$ is a factor $\sim 3$ less for the merger model than for the delayeddetonation model (see Sect. 2 and Table 1).

Pakmor et al. (2013) suggest that all SNe Ia derive from mergers of two WDs, except for pure deflagrations in near$\mathrm{M}_{\mathrm{Ch}}$ WDs that leave bound remnants behind - a model that matches the observables of SN 2002cx-like SNe well (see Phillips et al. 2007; Kromer et al. 2013). We therefore also include the N5def model of Fink et al. (2013).

\section{Results}

In Table 1, we have compiled a selection of [Mn/Fe] yields for different supernova types from the literature. It is evident that currently only models involving thermonuclear explosions of near- $\mathrm{M}_{\mathrm{Ch}}$ WDs predict $[\mathrm{Mn} / \mathrm{Fe}]>0.0$. Assuming that we are not missing a significant nucleosynthetic production site of $\mathrm{Mn}$, this alone already tells us that near- $\mathrm{M}_{\mathrm{Ch}}$ WDs primaries must contribute significantly to the production of $\mathrm{Mn}$ and $\mathrm{Fe}$, and therefore constitute a significant fraction of SNe Ia. To corroborate this result and to place further constraints on the relative fractions of near- $\mathrm{M}_{\mathrm{Ch}}$ and sub- $\mathrm{M}_{\mathrm{Ch}}$ WD primaries, we consider five

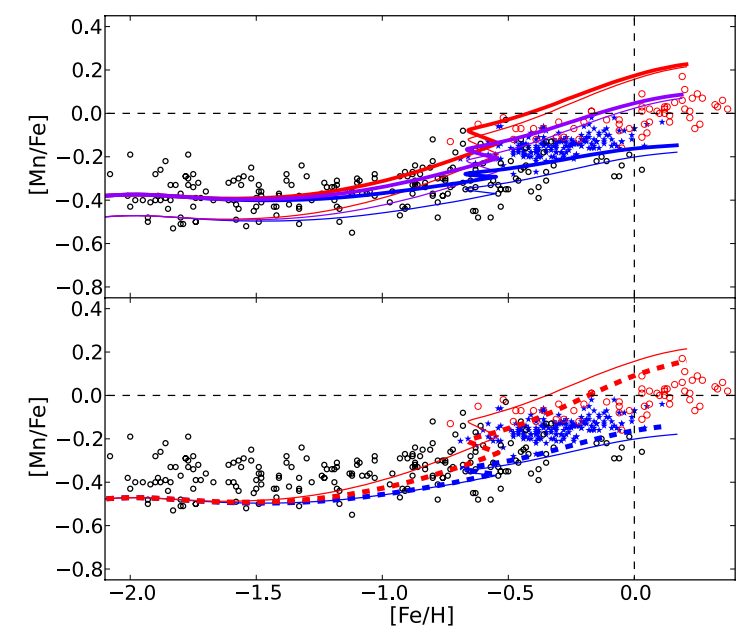

Fig. 1. $[\mathrm{Mn} / \mathrm{Fe}]$ vs. $[\mathrm{Fe} / \mathrm{H}]$ in the solar vicinity. Open black squares are data from Sobeck et al. (2006), blue stars are from Reddy et al. (2003), and red open dots are thin-disc data from Feltzing et al. (2007). Top panel: thin lines are for massive star yields from Woosley \& Weaver (1995), thick lines enhanced their Mn yields by 25 per cent. Red lines are for case $M_{C h}$, blue lines for case sub- $M_{C h}$, and case mix are the purple lines. Bottom panel: dashed thick blue line is for case sub$M_{C h}+2002 c x$, dashed thick red line is for case $M_{C h}+$. Thin blue and red lines are as in the top panel.

different chemical evolution cases, each case only differing in the nucleosynthetic yields assumed for SN Ia as listed here:

- case $M_{C h}$ : SN Ia yields are from the N100 model of a delayed detonation in a near- $\mathrm{M}_{\mathrm{Ch}} \mathrm{WD}$ (Seitenzahl et al. 2013).

- case sub-M $M_{C h}$ : SN Ia yields are from the violent merger of a 1.1 with a $0.9 M_{\odot}$ WD (Pakmor et al. 2012).

- case mix: $50 \%$ of SNe Ia explode as in case $M_{C h}$ and $50 \%$ as in case sub- $M_{C h}$.

- case $M_{C h}+$ : similar to case $M_{C h}$, but SN Ia yields depend on progenitor metallicity (using models N100_Z0.01, N100_Z0.1, and N100 from Seitenzahl et al. 2013).

- case sub- $M_{C h}+2002$ cx: $20 \%$ of SNe Ia explode as pure deflagrations leaving remnants (model N5def from Kromer et al. 2013), and the remaining $80 \%$ explode as in case $s u b-M_{C h}$.

In Fig. 1 (top), we compare the results of the chemical evolution calculations for $[\mathrm{Mn} / \mathrm{Fe}]$ of case $M_{C h}$, case $s u b-M_{C h}$, and case mix to observational data from the Galaxy. In addition to the standard yields from Woosley \& Weaver (1995) (which trace the data along the lower edge at $[\mathrm{Fe} / \mathrm{H}] \lesssim-1.0)$, we also include evolution models with their Mn yield enhanced by 25 per cent. These Mn-enhanced models demonstrate that the final $\mathrm{Mn}$ at high metallicity is rather insensitive to the assumed massive star yields at low metallicity. Naturally, owing to the sub-solar production ratio of $[\mathrm{Mn} / \mathrm{Fe}]$ of sub- $\mathrm{M}_{\mathrm{Ch}}$-based $\mathrm{SNe}$ Ia explosions, case sub- $M_{C h}$ falls short of reproducing the observed trend. The results of case $M_{C h}$ on the other hand reach and actually exceed the solar abundance. The data are best reproduced by a scenario where both sub- $\mathrm{M}_{\mathrm{Ch}}$ and near- $\mathrm{M}_{\mathrm{Ch}}$ primaries are present at roughly equal proportions. These results are a clear indication that $\mathrm{SNe}$ Ia cannot exclusively stem from sub-M $\mathrm{M}_{\mathrm{Ch}}$ WD primaries, owing to their inability to produce enough $\mathrm{Mn}$, as compared to the solar abundance.

In Fig. 1 (bottom), we show the results of the chemical evolution calculations for $[\mathrm{Mn} / \mathrm{Fe}]$ of case $M_{C h}+$ and case sub$M_{C h}+2002 c x$. It is evident that using the metallicity-dependent yields reduces $[\mathrm{Mn} / \mathrm{Fe}]$ somewhat, but the effect is secondary. In light of Pakmor et al. (2013), we note that case $s u b-M_{C h}+2002 c x$ also falls significantly short of reaching solar $[\mathrm{Mn} / \mathrm{Fe}]$, even 
though case sub- $M_{C h}+2002 c x$ assumes a very high fraction of 2002cx-like SNe. The expected relative fraction SN 2002cxlike $\mathrm{SNe}$ is around 4 per cent, Li et al. 2011. Although model N5def almost has the same $[\mathrm{Mn} / \mathrm{Fe}]$ production factor as the $\mathrm{N} 100$ model, it produces much less $\mathrm{Fe}$ and $\mathrm{Mn}$ in total (a factor $\sim 3.5$ less, which is expeced to be typical for the faint SN 2002cx-like objects), which explains its relatively small impact on $[\mathrm{Mn} / \mathrm{Fe}]$.

\section{Conclusions}

The observed abundance trend of $[\mathrm{Mn} / \mathrm{Fe}]$ at $[\mathrm{Fe} / \mathrm{H}] \gtrsim 0.0$ suggests that sub- $\mathrm{M}_{\mathrm{Ch}}$ WD primaries cannot be the only progenitors producing $\mathrm{SNe} I \mathrm{I}$ in the Galaxy; either only near- $\mathrm{M}_{\mathrm{Ch}}$ primary WDs or a combination of near- $\mathrm{M}_{\mathrm{Ch}}$ and sub- $\mathrm{M}_{\mathrm{Ch}}$ primaries (a mix of equal parts results in a good match to data) is needed to reach the observed $[\mathrm{Mn} / \mathrm{Fe}]$ in the Sun. Matteucci et al. (2009) reaches a similar conclusion. They find that to reproduce $[\mathrm{O} / \mathrm{Fe}]$ as a function of $[\mathrm{Fe} / \mathrm{H}]$ and the metallicity distribution of G-type stars in the solar neighbourhood, both SDS and DDS progenitors must contribute to the Galactic population of SNe Ia. Based on our chemical evolution calculations, we can also exclude that a combination of sub- $\mathrm{M}_{\mathrm{Ch}}$ WD primaries and near- $\mathrm{M}_{\mathrm{Ch}}$ WD primaries exploding as pure deflagrations that only partially unbind the primary (i.e. $2002 \mathrm{cx}$-like $\mathrm{SNe}$ ) constitute the entirety of SN Ia progenitors.

We speculate that the discrepancy between the chemical evolution of $\mathrm{Mn}$ in dwarf spheroidal galaxies (dSph) and in the Milky Way (see McWilliam et al. 2003; North et al. 2012) could also be explained if SNe Ia did not arise from a unique channel. A different relative frequency of near- $\mathrm{M}_{\mathrm{Ch}}$ and sub- $\mathrm{M}_{\mathrm{Ch}}$ primaries (e.g. due to star formation history or metallicity) could also be a solution to the $\mathrm{Mn}$ problem in $\mathrm{dSph}$, since this would have an overall similar effect to the strong intrinsic dependency on metallicity of the Mn yields invoked by Cescutti et al. (2008). In closing, we caution that any effect that raises $[\mathrm{Mn} / \mathrm{Fe}]$ for sub$\mathrm{M}_{\mathrm{Ch}}$ primary explosion models to super-solar would remove the need for a large portion of near- $\mathrm{M}_{\mathrm{Ch}}$ primaries.

Acknowledgements. I.R.S. was funded by the Deutsche Forschungsgemeinschaft (DFG) through the graduate school of "Theoretical Astrophysics and Particle Physics" (GRK 1147). F.K.R. was supported by the DFG via the Emmy Noether Programme (RO 3676/1-1) and by the ARCHES prize of the German Federal Ministry of Education and Research (BMBF), and R.P. by the European Research Council under ERC-StG grant EXAGAL-308037. The DAAD/Go8 German-Australian exchange programme provided funding for collaboration.

\section{References}

Asplund, M., Grevesse, N., Sauval, A. J., \& Scott, P. 2009, ARA\&A, 47, 481 Bildsten, L., \& Hall, D. M. 2001, ApJ, 549, L219

Bravo, E., \& García-Senz, D. 2008, A\&A, 478, 843

Bravo, E., \& García-Senz, D. 2009, ApJ, 695, 1244

Bravo, E., \& Martínez-Pinedo, G. 2012, Phys. Rev. C, 85, 055805

Bravo, E., Isern, J., Canal, R., \& Labay, J. 1992, A\&A, 257, 534

Cescutti, G., Matteucci, F., Lanfranchi, G. A., \& McWilliam, A. 2008, A\&A, 491, 401

Chiappini, C., Matteucci, F., \& Gratton, R. 1997, ApJ, 477, 765

Dan, M., Rosswog, S., Guillochon, J., \& Ramirez-Ruiz, E. 2011, ApJ, 737, 89

Dessart, L., Burrows, A., Ott, C. D., et al. 2006, ApJ, 644, 1063

Feltzing, S., Fohlman, M., \& Bensby, T. 2007, A\&A, 467, 665

Fink, M., Röpke, F. K., Hillebrandt, W., et al. 2010, A\&A, 514, A53

Fink, M., Kromer, M., Seitenzahl, I. R., et al. 2013, MNRAS, submitted [arXiv: 1308.3257]

François, P., Matteucci, F., Cayrel, R., et al. 2004, A\&A, 421, 613

Gamezo, V. N., Khokhlov, A. M., \& Oran, E. S. 2005, ApJ, 623, 337

Gratton, R. G. 1989, A\&A, 208, 171

Gratton, R. G., \& Sneden, C. 1988, A\&A, 204, 193

Gratton, R. G., \& Sneden, C. 1991, A\&A, 241, 501

Greggio, L. 2005, A\&A, 441, 1055

Greggio, L., \& Renzini, A. 1983, A\&A, 118, 217
Grevesse, N., Asplund, M., Sauval, A. J., \& Scott, P. 2010, Ap\&SS, 328, 179 Hillebrandt, W., Kromer, M., Röpke, F. K., \& Ruiter, A. J. 2013, Frontiers of Physics, 8, 116

Iben, Jr., I., \& Tutukov, A. V. 1984, ApJS, 54, 335

Iwamoto, K., Brachwitz, F., Nomoto, K., et al. 1999, ApJS, 125, 439

Jordan, G. C., Gupta, S. S., \& Meyer, B. S. 2003, Phys. Rev. C, 68, 065801

Jordan, IV, G. C., Graziani, C., Fisher, R. T., et al. 2012a, ApJ, 759, 53

Jordan, IV, G. C., Perets, H. B., Fisher, R. T., \& van Rossum, D. R. 2012b, ApJ, 761, L23

Kasen, D., Röpke, F. K., \& Woosley, S. E. 2009, Nature, 460, 869

Kobayashi, C., \& Nomoto, K. 2009, ApJ, 707, 1466

Kobayashi, C., Umeda, H., Nomoto, K., Tominaga, N., \& Ohkubo, T. 2006, ApJ, 653,1145

Kobayashi, C., Karakas, A. I., \& Umeda, H. 2011, MNRAS, 414, 3231

Kromer, M., Sim, S. A., Fink, M., et al. 2010, ApJ, 719, 1067

Kromer, M., Fink, M., Stanishev, V., et al. 2013, MNRAS, 429, 2287

Li, W., Chornock, R., Leaman, J., et al. 2011, MNRAS, 412, 1473

Limongi, M., \& Chieffi, A. 2003, ApJ, 592, 404

Ma, H., Woosley, S. E., Malone, C. M., Almgren, A., \& Bell, J. 2013, ApJ, 771, 58

Maeda, K., Röpke, F. K., Fink, M., et al. 2010, ApJ, 712, 624

Maoz, D., \& Mannucci, F. 2012, PASA, 29, 447

Matteucci, F., \& Greggio, L. 1986, A\&A, 154, 279

Matteucci, F., Spitoni, E., Recchi, S., \& Valiante, R. 2009, A\&A, 501, 531

Mazzali, P. A., Röpke, F. K., Benetti, S., \& Hillebrandt, W. 2007, Science, 315, 825

McWilliam, A., Rich, R. M., \& Smecker-Hane, T. A. 2003, ApJ, 592, L21

Meakin, C. A., Seitenzahl, I., Townsley, D., et al. 2009, ApJ, 693, 1188

Mochkovitch, R., \& Livio, M. 1990, A\&A, 236, 378

Nomoto, K., \& Kondo, Y. 1991, ApJ, 367, L19

Nomoto, K., Tominaga, N., Umeda, H., Kobayashi, C., \& Maeda, K. 2006, Nucl. Phys. A, 777, 424

North, P., Cescutti, G., Jablonka, P., et al. 2012, A\&A, 541, A45

Pakmor, R., Kromer, M., Röpke, F. K., et al. 2010, Nature, 463, 61

Pakmor, R., Hachinger, S., Röpke, F. K., \& Hillebrandt, W. 2011, A\&A, 528, A117

Pakmor, R., Kromer, M., Taubenberger, S., et al. 2012, ApJ, 747, L10

Pakmor, R., Kromer, M., Taubenberger, S., \& Springel, V. 2013, ApJ, 770, L8

Parikh, A., José, J., Seitenzahl, I. R., \& Röpke, F. K. 2013, A\&A, 557, A3

Phillips, M. M., Li, W., Frieman, J. A., et al. 2007, PASP, 119, 360

Piersanti, L., Gagliardi, S., Iben, Jr., I., \& Tornambé, A. 2003a, ApJ, 598, 1229

Piersanti, L., Gagliardi, S., Iben, Jr., I., \& Tornambé, A. 2003b, ApJ, 583, 885

Pignatari, M., Herwig, F., Hirschi, R., et al. 2013, ApJS, submitted [arXiv: 1307.6961]

Plewa, T. 2007, ApJ, 657, 942

Raskin, C., Scannapieco, E., Fryer, C., Rockefeller, G., \& Timmes, F. X. 2012, ApJ, 746, 62

Reddy, B. E., Tomkin, J., Lambert, D. L., \& Allende Prieto, C. 2003, MNRAS, 340,304

Röpke, F. K., \& Niemeyer, J. C. 2007, A\&A, 464, 683

Röpke, F. K., Woosley, S. E., \& Hillebrandt, W. 2007, ApJ, 660, 1344

Röpke, F. K., Kromer, M., Seitenzahl, I. R., et al. 2012, ApJ, 750, L19

Ruiter, A. J., Belczynski, K., \& Fryer, C. 2009, ApJ, 699, 2026

Ruiter, A. J., Belczynski, K., Sim, S. A., et al. 2011, MNRAS, 1282

Ruiter, A. J., Sim, S. A., Pakmor, R., et al. 2013, MNRAS, 429, 1425

Saio, H., \& Nomoto, K. 1985, A\&A, 150, L21

Saio, H., \& Nomoto, K. 1998, ApJ, 500, 388

Saio, H., \& Nomoto, K. 2004, ApJ, 615, 444

Seitenzahl, I. R., Taubenberger, S., \& Sim, S. A. 2009, MNRAS, 400, 531

Seitenzahl, I. R., Ciaraldi-Schoolmann, F., \& Röpke, F. K. 2011, MNRAS, 414, 2709

Seitenzahl, I. R., Ciaraldi-Schoolmann, F., Röpke, F. K., et al. 2013, MNRAS, 429, 1156

Sim, S. A., Röpke, F. K., Hillebrandt, W., et al. 2010, ApJ, 714, L52

Sobeck, J. S., Ivans, I. I., Simmerer, J. A., et al. 2006, AJ, 131, 2949

Stritzinger, M., Leibundgut, B., Walch, S., \& Contardo, G. 2006, A\&A, 450, 241

Summa, A., Ulyanov, A., Kromer, M., et al. 2013, A\&A, 554, A67

Thielemann, F.-K., Nomoto, K., \& Yokoi, K. 1986, A\&A, 158, 17

Timmes, F. X. 1994, ApJ, 423, L131

Timmes, F. X., Woosley, S. E., \& Weaver, T. A. 1995, ApJS, 98, 617

Truran, J. W., Arnett, W. D., \& Cameron, A. G. W. 1967, Can. J. Phys., 45, 2315

Tutukov, A. V., \& Yungelson, L. R. 1979, Acta Astron., 29, 665

Webbink, R. F. 1984, ApJ, 277, 355

Whelan, J., \& Iben, I. J. 1973, ApJ, 186, 1007

Woosley, S. E., \& Weaver, T. A. 1994, ApJ, 423, 371

Woosley, S. E., \& Weaver, T. A. 1995, ApJS, 101, 181

Woosley, S. E., Arnett, W. D., \& Clayton, D. D. 1973, ApJS, 26, 231

Yoon, S.-C., Podsiadlowski, P., \& Rosswog, S. 2007, MNRAS, 380, 933 